\title{
Thermography as a non-ionizing quantitative tool for diagnosing periapical inflammatory lesions
}

\author{
M. Atef Aboushady ${ }^{1,2}$, Wael Talaat ${ }^{3,4,5^{*}} \mathbb{D}$, Zaid Hamdoon ${ }^{3}$, Tarek M.Elshazly ${ }^{2}$, Nivin Ragy ${ }^{6}$, \\ Christoph Bourauel ${ }^{2+}$ and Sameh Talaat ${ }^{2,7+}$
}

\begin{abstract}
Background: Thermography is a contemporary imaging modality based on acquiring and analyzing thermal data using non-contact devices. The aim of the present study was to assess the validity of thermography, compared with that of the reference-standard, for the diagnosis of periapical inflammatory lesions and to evaluate the temperature ranges for acute pulpitis with apical periodontitis (AAP), acute periapical abscess (AA) and chronic periapical abscess (CA).
\end{abstract}

Methods: AAP, AA and CA were diagnosed based on clinical and radiographic criteria. Thermographic data were acquired using the FLIR E-5 Infrared Camera. Extraoral thermal images were taken from the front and right and left sides of patients whose mouths were closed, and one intraoral thermal image was taken from the palatal perspective. Agreement in the diagnoses based on the combination of clinical and radiographic assessments and the thermographic evaluation was calculated. The temperature ranges of the three diagnostic subgroups were also measured.

Results: A total of 80 patients were enrolled in this study. The mean intraoral thermal image temperature for AA was 37.26 \pm 0.36 , that for CA was $35.03 \pm 0.63$ and that for AAP was $36.07 \pm 0.45$. The differences between the mean intraoral thermal temperatures of the three diagnostic groups were statistically significant $(P<0.001)$. The result of the Kappa coefficient of agreement between the combination of clinical and radiographic assessments and the thermographic evaluation was significant $(P<0.001)$.

Conclusions: Thermography is an effective, quantitative and nonionizing approach that can be used for the diagnosis of periapical inflammatory lesions. The results of the present study indicated that the highest thermal image temperatures were recorded for AA. Thermography might be able to detect inflammatory reactions during the preclinical stage, leading to early diagnosis.

Keywords: Thermography, Acute pulpitis with apical periodontitis, Acute periapical abscess, Chronic periapical abscess, Periapical inflammatory intraoral lesions

*Correspondence: wtaha@sharjah.ac.ae

${ }^{\dagger}$ Christoph Bourauel and Sameh Talaat have equally contributed to this work

${ }^{3}$ Department of Oral and Craniofacial Health Sciences, College of Dental Medicine, University of Sharjah, Sharjah 27272, UAE

Full list of author information is available at the end of the article

\section{Background}

Periapical inflammatory lesions result from pulpal lesions or disrupted periodontal attachments that extend to the apex, furcation or lateral canals. Periapical lesions are usually consequences of the vascular and anatomic connections between the pulp and periodontium. A thorough history and careful clinical and radiographic 
examinations are required to diagnose such lesions and develop a treatment plan. However, the diagnosis of periapical inflammatory lesions is always a challenge, even for experienced dental practitioners, due to the multifactorial origin of these lesions, which include diverse interactions between different strains and species of microbes [1].

Thermography is a contemporary imaging modality with various potential applications in dentistry. The technology is based on the acquisition and analysis of thermal data using noncontact devices, and it depends on infrared electromagnetic radiation, which is emitted by objects with temperatures above absolute zero [2]. Temperature differences can be charted on a two-dimensional image, and accordingly, the temperatures of individual spots in an area of interest at a certain time can be recorded [3].

Human tissues are ideal emitters of infrared radiation at room temperature. It has been reported that most of the infrared radiation emitted by the human body has long wavelengths $(8-15 \mu \mathrm{m})$. Infrared cameras can convert the emitted radiation into electrical signals, which can then be displayed using colors to represent temperature values. Ultimately, a quantitative temperature map of the area of interest is produced, and this map can be utilized to identify different pathological conditons [4].

Thermography has been used in different applications in dentistry. Chronic orofacial pain and temporomandibular disorders were successfully classified thermally $[5,6]$. Thermography has been used to evaluate thermal insults to the dental pulp. Thermal image analysis showed that the pulpal temperature increased significantly following the electrothermal debonding of orthodontic brackets, confirming the high risk to the pulp [7]. Thermophotonic lock-in imaging using photothermal wave principles has been used to detect early dental carious lesions [8]. Moreover, thermography has been used successfully to detect malignancies of human hard and soft tissues [9, $10]$.

The aims of the present study were to assess the validity of thermography for the diagnosis of periapical inflammatory lesions and to compare it with the reference-standard, X-ray radiographs. The temperature ranges for acute pulpitis with apical periodontitis lesions and acute and chronic periapical abscess lesions were evaluated.

\section{Methods}

The study was conducted on patients who were treated at the Oral Diagnosis Clinic at the Faculty of Oral and Dental Medicine, Future University, Egypt, between September 2017 and March 2020. All the patients underwent comprehensive dental, intraoral, and extraoral diagnostic examination procedures. Patients were included in the study if they had any signs or symptoms of swelling, tenderness with palpation, pain with percussion, widening of the lamina dura or apical radiolucency related to any maxillary tooth except the third molars. The exclusion criteria included an age under 18 years or over 60 years; inflammatory conditions of the oral mucosa; severe cognitive, mental and locomotive deficiencies; physiological or pathological conditions associated with changes in thermal homeostasis; smoking habits and the use of nonsteroidal anti-inflammatory drugs. All the patients were informed of the aim of the study, and they signed a formal consent form before participation. Ethical approval for this study was obtained from the Research Ethics Committee at Future University, Egypt. The guidelines of the Declaration of Helsinki were followed in this investigation.

AAP, AA and CA were diagnosed based on clinical and radiographic criteria (Table 1). The inflammatory lesions were related to maxillary teeth except the third molars. The clinical examination involved the observation of decayed and broken restorations, fracture lines, swelling and sinus tracks. Tenderness of the apical area upon palpation, percussion tests, pulp sensitivity tests and tooth mobility evaluations were also performed. The radiographic diagnosis was based on the identification of areas of radiolucency on periapical radiographs. The clinical and radiographic examinations were performed by two calibrated investigators with over 10 years of clinical experience.

Table 1 The clinical and radiographic criteria used to diagnose AA, CA and AAP

\begin{tabular}{|c|c|c|c|}
\hline & AA & CA & AAP \\
\hline Clinical criteria & $\begin{array}{l}\text { Throbbing and pain to light pressure, bit- } \\
\text { ing, touching and percussion accom- } \\
\text { panied by tenderness to palpation and } \\
\text { mobility of the tooth. Intra-oral and/or } \\
\text { extra-oral swelling may be present }\end{array}$ & $\begin{array}{l}\text { Presence of a draining sinus on the oral } \\
\text { mucosa or occasionally on the facial } \\
\text { skin not usually associated with pain }\end{array}$ & $\begin{array}{l}\text { Spontaneous pain, extreme sensitivity to } \\
\text { heat and cold, awakening at night and } \\
\text { considerable tenderness to percussion } \\
\text { and pressure on the tooth }\end{array}$ \\
\hline Radiographic criteria & $\begin{array}{l}\text { No evidence of periapical changes or a } \\
\text { slight thickening of the periodontal } \\
\text { ligament space }\end{array}$ & $\begin{array}{l}\text { Periapical radiolucent area related to the } \\
\text { apex of the tooth }\end{array}$ & $\begin{array}{l}\text { Widening of the periodontal ligament } \\
\text { space and loss of lamina dura around } \\
\text { the apex }\end{array}$ \\
\hline
\end{tabular}




\section{Thermographic investigation}

Thermographic data were acquired using the FLIR E-5 Infrared Camera with MSX (FLIR Systems, Oregon, USA). Images were stored on a memory card in the TIFF file format and processed using specialized software (FLIR Tools Thermal Analysis and Reporting; Desktop, FLIR Systems, Oregon, USA). The camera was calibrated considering the emissivity parameters of the human body $\left(\varepsilon=0.98\right.$ and $\left.\lambda^{\prime} 2 \mu \mathrm{m}\right)$ and the ambient conditions (humidity and temperature), and a thermal range from 20 to $250{ }^{\circ} \mathrm{C} \pm 2{ }^{\circ} \mathrm{C}$, a pixel infrared resolution of $10,800(120 \times 90)$, and adequate shade were selected for optimal color registration. A standard protocol was used by positioning all the electronic devices at least $2 \mathrm{~m}$ from the patient, covering windows and reflective surfaces with opaque dark textiles and turning off artificial light sources. Room temperature was set to $20 \pm 1{ }^{\circ} \mathrm{C}$, and the air currents were directed away from the patient and toward the periphery of the room. The patients were asked to refrain from smoking, eating and drinking for $60 \mathrm{~min}$ prior to the examination, and they remained at rest for $10 \mathrm{~min}$ before the procedure. The oral cavity was cooled by rinsing with cold water $\left(10^{\circ} \mathrm{C}\right)$ for $1 \mathrm{~min}$.

A profile-guided thermography scanner was designed to provide a guided trajectory for the infrared camera using a machined profile, which was designed as an offset contour of the human mandible to maintain the camera in a position such that it faced the patient. Bearing housing was designed to roll on the guide holding the camera, and upper and lower bearings were used to hold the positioning mechanism and the camera, while side bearings were used to adjust the camera angle to face the patient (Fig. 1).

Patients rinsed their mouths with cold water then three extraoral thermal view images were taken from the front and right and left sides of patients whose mouths were closed. The procedure was repeated for five times at oneminute intervals. One intraoral thermal image was then taken from the palatal perspective after $5 \mathrm{~min}$ at a distance of $20 \mathrm{~cm}$ (Fig. 2).

Thermographic data recording was performed by a third investigator who was blinded to the clinical and radiographic patient records. Agreement in the diagnoses of AAP, AA and CA based on the combination of clinical and radiographic assessments and the thermographic evaluation was calculated to assess the validity of thermography. The temperature ranges of the three diagnostic subgroups were also measured.

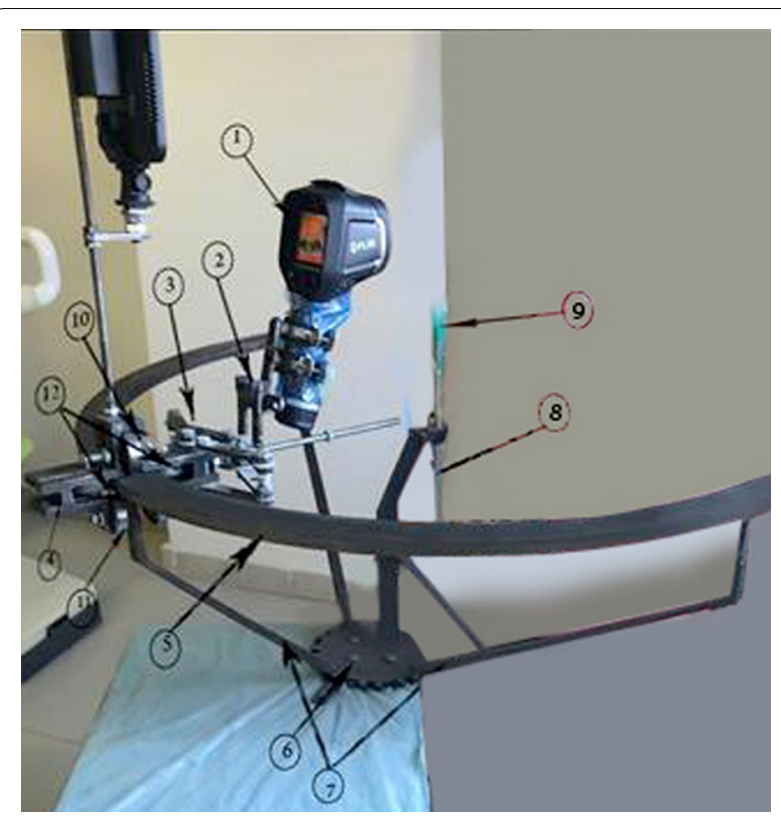

Fig. 1 Profile-guided thermography scanner showing: 1. Infrared camera, 2. Adjusting angles, 3. Scissors mechanism, 4. Bearing housing, 5. Guiding profile, 6. Fixing flange, 7. Guide supports, 8. Chin rest support, 9. Chin rest, 10. Upper bearing, 11. Lower bearings, 12. Side bearings

\section{Statistical analysis}

The data analysis was performed using the Statistical Package for Social Sciences (SPSS) version 16.0 (SPSS Inc., Chicago, IL, USA). The thermography results are expressed as the mean \pm standard deviation (SD). Analysis of variance (ANOVA) was used for testing the null hypothesis that the mean of the groups are equal, followed by the Bonferroni method for multiple comparisons. The Kappa coefficient of agreement was used to assess the relation between the combination of clinical and radiographic assessments and the thermographic evaluation following the conversion of the quantitative data to dichotomous categorical data. P-values of $<0.05$ were considered statistically significant.

\section{Results}

A total of 80 patients (42 females and 38 males) were enrolled in this study. Twenty-seven patients with a mean age of $37.37 \pm 9.97$ years were diagnosed with AA, 29 patients with a mean age of $39.1 \pm 12.34$ years were diagnosed with CA, and 24 patients with a mean age of $36.96 \pm 10.98$ years were diagnosed with AAP.

The mean intraoral thermal image temperature for AA was $37.26 \pm 0.36$, that for CA was $35.03 \pm 0.63$ and that for AAP was $36.07 \pm 0.45$. The rates of change in extraoral thermal temperatures with time for the 3 diagnostic groups are shown in Table 2 and Fig. 3 . The results 


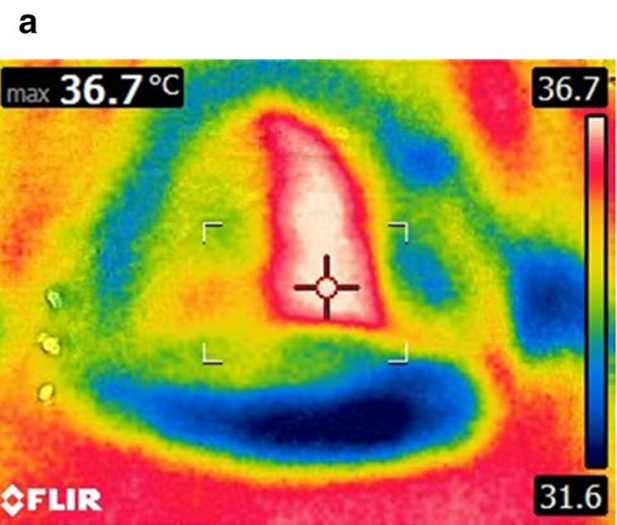

\section{b}

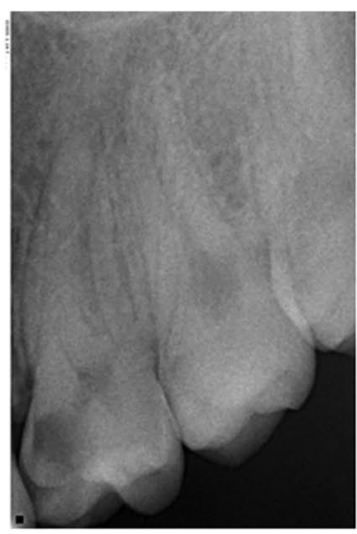

C

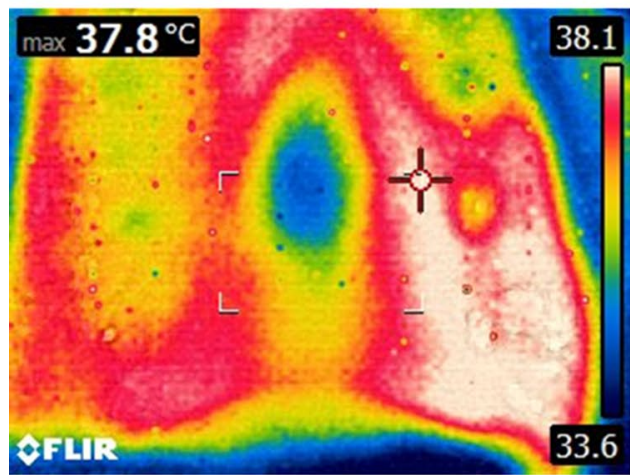

d

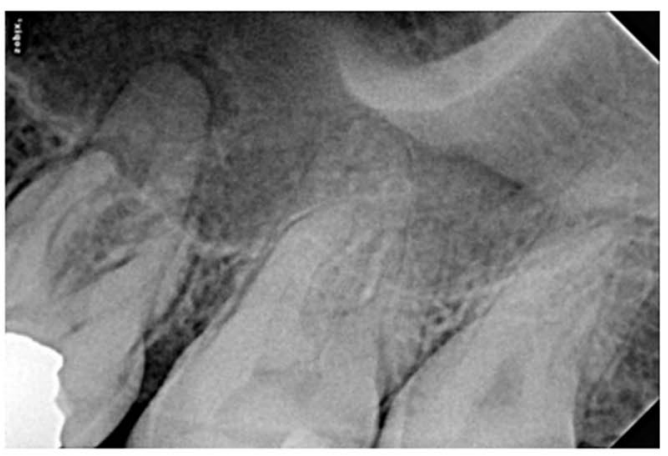

e

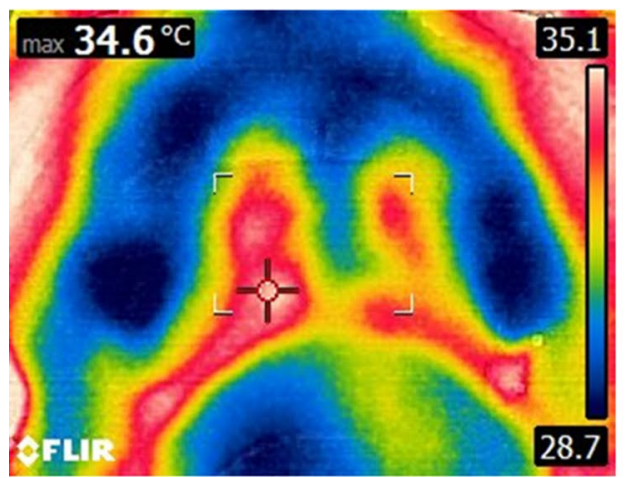

f

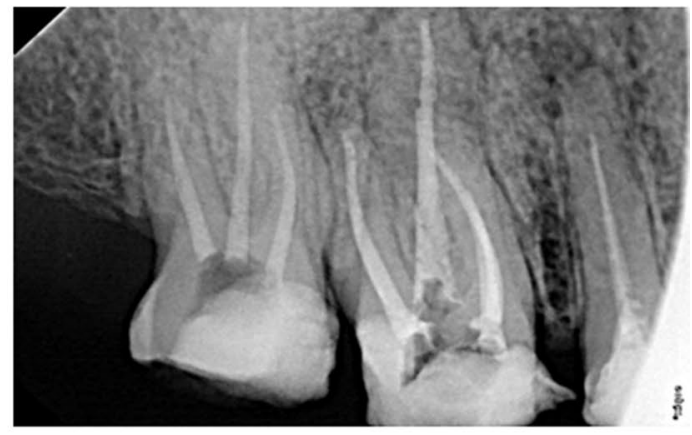

Fig. 2 a Thermal image for AAP related to the upper left first molar. The heat detector is pointing to the highest temperature $\left(36.7^{\circ} \mathrm{C}\right) . \mathbf{b}$ Periapical radiograph showing widening of the lamina dura around the mesial root of the same tooth. $\mathbf{c}$ Thermal image for AA related to the upper left first molar with the highest temperature $\left(37.8^{\circ} \mathrm{C}\right)$ detected using Flir Tools software. $\mathbf{d}$ Periapical radiograph showing widening of the lamina dura around the roots of the same tooth with radiolucency related to the apex of the roots. e Thermal image for CA related to the upper right second premolar. f Periapical radiograph showing radiolucency surrounding the apex of the upper right second premolar. A sinus tract related to the same tooth was detected on clinical examination

of the analysis of variance (ANOVA) for the assessment of between-group differences were statistically significant $(P<0.001)$. The differences between the mean thermal image temperatures of the three diagnostic groups were statistically significant $(P<0.001)$ (Table 3$)$.

The Kappa coefficient of agreement $(0.970)$ between the combination of clinical and radiographic assessments 
Table 2 Comparison of the rate of change in extraoral thermal temperatures with time for the three diagnostic groups

\begin{tabular}{|c|c|c|c|c|c|c|c|c|}
\hline & \multirow[t]{2}{*}{$\mathrm{N}$} & \multirow[t]{2}{*}{ Mean } & \multirow[t]{2}{*}{ SD } & \multirow[t]{2}{*}{ SEM } & \multicolumn{2}{|c|}{$95 \%$ confidence interval for mean } & \multirow[t]{2}{*}{ Minimum } & \multirow[t]{2}{*}{ Maximum } \\
\hline & & & & & Lower bound & Upper bound & & \\
\hline \multicolumn{9}{|l|}{$A A$} \\
\hline Temp. After 1 min & 81 & 35.44 & 0.64 & 0.07 & 35.30 & 35.58 & 33.90 & 37.02 \\
\hline Temp. After 2 min & 81 & 35.41 & 0.59 & 0.07 & 35.28 & 35.54 & 33.70 & 37.00 \\
\hline Temp. After 3 min & 81 & 35.65 & 0.61 & 0.07 & 35.52 & 35.79 & 33.90 & 37.50 \\
\hline Temp. After 4 min & 81 & 35.91 & 0.62 & 0.07 & 35.77 & 36.05 & 34.00 & 37.60 \\
\hline Temp. After 5 min & 81 & 36.21 & 0.71 & 0.08 & 36.06 & 36.37 & 34.10 & 37.80 \\
\hline \multicolumn{9}{|l|}{$C A$} \\
\hline Temp. After 1 min & 87 & 33.76 & 1.32 & 0.14 & 33.48 & 34.04 & 27.10 & 35.82 \\
\hline Temp. After 2 min & 87 & 33.70 & 1.34 & 0.14 & 33.42 & 33.99 & 27.10 & 35.70 \\
\hline Temp. After 3 min & 87 & 33.97 & 1.08 & 0.12 & 33.74 & 34.21 & 27.80 & 35.80 \\
\hline Temp. After 4 min & 87 & 34.29 & 0.78 & 0.08 & 34.12 & 34.45 & 31.30 & 35.90 \\
\hline Temp. After 5 min & 87 & 34.55 & 0.71 & 0.08 & 34.40 & 34.70 & 32.50 & 36.10 \\
\hline \multicolumn{9}{|l|}{ AAP } \\
\hline Temp. After 1 min & 72 & 34.57 & 0.69 & 0.08 & 34.41 & 34.73 & 32.80 & 35.88 \\
\hline Temp. After 2 min & 72 & 34.52 & 0.67 & 0.08 & 34.37 & 34.68 & 32.90 & 35.90 \\
\hline Temp. After 3 min & 72 & 34.79 & 0.63 & 0.07 & 34.65 & 34.94 & 33.50 & 36.10 \\
\hline Temp. After 4 min & 72 & 35.05 & 0.61 & 0.07 & 34.91 & 35.19 & 33.60 & 36.60 \\
\hline Temp. After 5 min & 72 & 35.30 & 0.59 & 0.07 & 35.16 & 35.44 & 33.90 & 37.10 \\
\hline
\end{tabular}

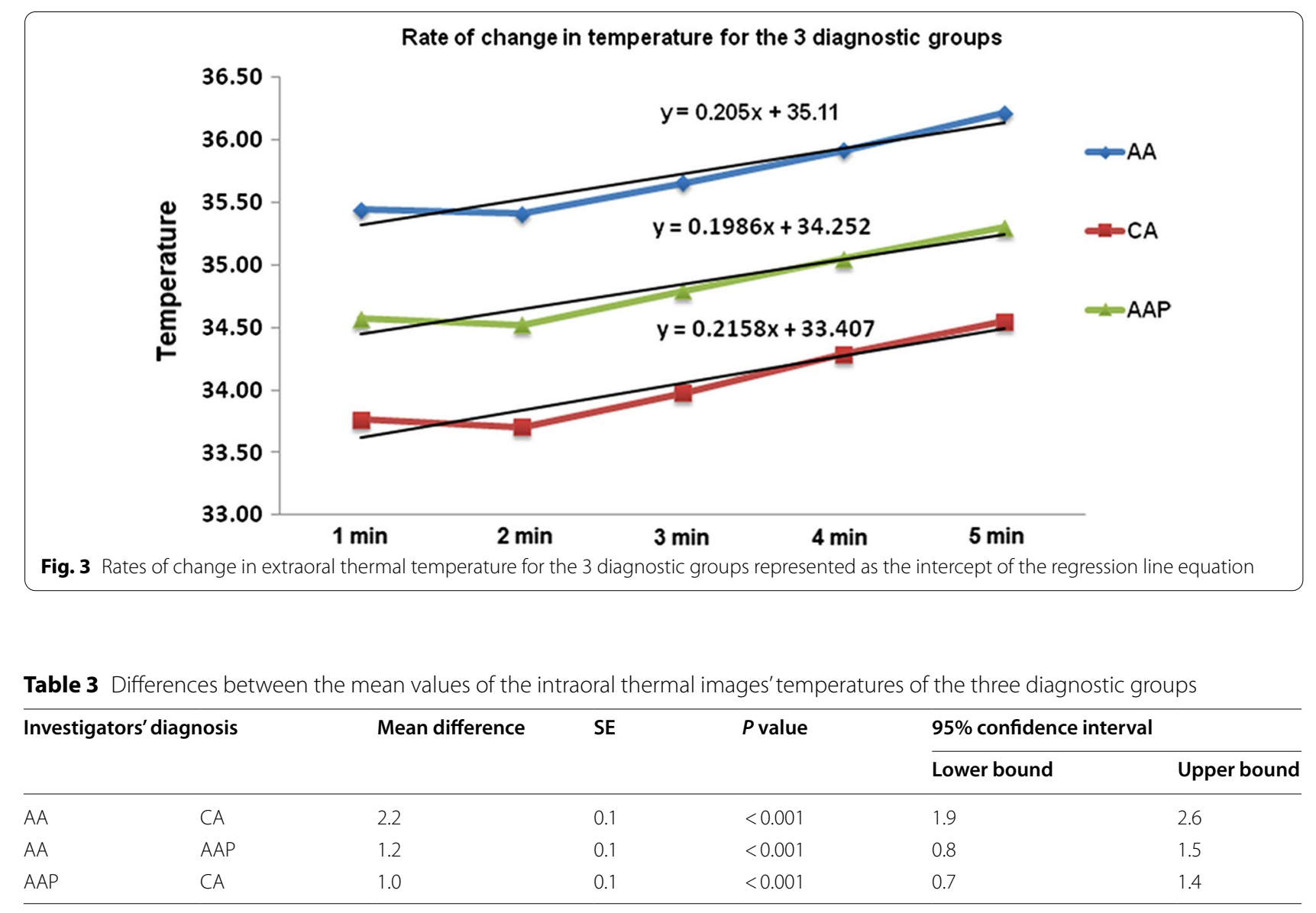


and the thermographic evaluation was significant $(P<0.001)$.

\section{Discussion}

Thermography is an imaging modality with a promising future in dental medicine. There are many advantages of thermography, including the possibility of evaluating different diseases without the use of ionizing radiation, which prevents many of the harmful effects of radiation and allows disease evaluation during pregnancy. In addition, thermography is a noncontact technology and is optimal for infection control. Thermography allows the comparison of different areas of interest on two-dimensional images and allows the evaluation to be conducted in real time [11]. The concept behind the use of thermography to aid in the diagnosis of pathologies is based on the fact that heat is always generated in response to inflammation, and thermography images are able to quantify the surface temperature, thus providing an assessment of the microcirculation in a specific area. It has been reported that a temperature difference of more than $3{ }^{\circ} \mathrm{C}$ usually indicates infection [12]. Furthermore, thermography is able to distinguish between different infections based on the range of temperature increases associated with each [13]. In the present study, the temperature ranges for each diagnostic group were identified, and the differences between the mean temperatures of the three diagnostic groups were statistically significant. In another study that evaluated the thermal image temperatures for facial cellulitis and dental abscess, a significant temperature difference between the two pathologic conditions was observed. The study reported the temperature differences between affected and contra-lateral unaffected side for abscess as $1.49 \pm 1.0$ and for cellulitis as $2.4 \pm 1.9$. The higher differences in facial cellulitis compared to dental abscess were attributed to the wider spread and more tissue damage caused by cellulitis. However, the authors reported a small sample size as a limitation of their study [14]. Other studies reported the efficiency of thermography in classifying chronic orofacial pain and in diagnosing temporomandibular disorders. The thermal classification of orofacial pain resulted in $92 \%$ agreement with the clinical diagnosis [5]. Internal derangement and osteoarthritis of the temporomandibular joint showed a temperature increase of $+0.4{ }^{\circ} \mathrm{C}$ compared to normal joints [6]. Thermography was also useful to confirm the thermal risk associated with the electro-thermal debonding of orthodontic brackets which resulted in an increase of the temperature of the pulp from 16.8 to $45.6{ }^{\circ} \mathrm{C}$ [7]. Inferior alveolar nerve deficit was successfully evaluated thermographically as it showed a temperature increase of $+0.5^{\circ} \mathrm{C}$ when compared to normal controls [5].
The capability of thermography for distinguishing the temperature ranges of different pathological conditions can be attributed to recent advancements in technology. The new generation of high-resolution cameras has the ability to detect even minor temperature changes resulting from different pathological conditions. These changes can result from the distinctive pathophysiological pathways of the different pathologies, which might still have a general inflammatory origin. At the beginning of the inflammatory process, the somatic-vegetative reflex of the adrenal gland is activated. This is usually followed by the secretion of histamine and kinins, which lead to local decreases in vascular resistance. Liver metabolic processes then produce acute phase proteins and clotting cascade proteins, leading to muscle protein proteolysis and fever. Increased permeability of the blood vessel endothelium, which leads to a movement of water to the perivascular space, usually occurs within the first hour following stimulation [15]. Edema and reddening usually follow due to the difficulty of capillary blood outflow from the site of inflammation, leading to passive hyperemia. The secretion of endogenic pyrogens such as interleukin 1 and tumor necrosis factor, excitation of the sympathetic system and increase in adrenal glucocorticoid levels lead to a febrile state. These changes, together with changes in the metabolic state of the inflammatory site, lead to a local increase in temperature [16].

The gradual increase in thermographic temperatures has been shown to be linked to the gradual reduction in the cooling effect of rinsing with cold water. After $5 \mathrm{~min}$, the thermographic temperatures are expected to reflect the increase in the concentrations of inflammatory mediators, which peak $96 \mathrm{~h}$ after the start of inflammation [17]. The results of the present study support the hypothesis that thermography might be able to detect the inflammatory reaction during the preclinical stage, leading to early diagnosis [18]. Thus, digital infrared imaging may be able to promote the early diagnosis of inflammatory conditions, even before clinical symptoms are observed [19].

The reported control temperature for the maxillary oral mucosa was $32.2{ }^{\circ} \mathrm{C}$, which makes it unlikely to have false positive results considering the results of the present study [20]. However, the use of thermography in dentistry still suffers from several limitations including the high price range for quality cameras, the inaccurate results in patients suffering from sunburns and facial scars and the technique sensitivity, which results in reduction in the images resolution if the angle and the distance are not adjusted [20]. 


\section{Conclusions}

Thermography is an effective, quantitative and nonionizing approach that can be used for the diagnosis of AAP, AA, CA. The results of the present study indicated that the highest thermal image temperatures were recorded for AA. Thermography might be able to detect inflammatory reactions during the preclinical stage, leading to early diagnosis.

\section{Abbreviations}

AAP: Acute pulpitis with apical periodontitis; AA: Acute periapical abscess; CA: Chronic periapical abscess.

\section{Acknowledgements}

Not applicable.

\section{Authors' contributions}

Study conception and design: MAA, CB, ST; data collection: MAA, WT, ZH, TME, $\mathrm{NR}, \mathrm{CB}, \mathrm{ST}$; analysis and interpretation of results: MAA, WT, ZH, TME, NR, CB, ST; draft manuscript preparation: MAA, WT, ZH, TME, NR, CB, ST. All authors reviewed the results and approved the final version of the manuscript.

\section{Funding}

This research did not receive any specific grant from funding agencies in the public, commercial, or not-for-profit sectors.

\section{Availability of data and materials}

The datasets used and/or analysed during the current study are available from the corresponding author on reasonable request.

\section{Declarations}

\section{Ethics approval and consent to participate}

This study was performed in line with the principles of the Declaration of Helsinki. Approval was granted by the Research Ethics Committee at the Future University in Egypt, Cairo, Egypt (FUE.REC (15)/12-2017). Written informed consents were obtained from all individual participants included in the study.Consent for publication.Consent for publication was obtained from all relevant individuals.

\section{Competing interests}

The authors declare that they have no competing interests.

\section{Author details}

${ }^{1}$ Department of Endodontics, Faculty of Oral and Dental Medicine, Future University in Egypt, Cairo, Egypt. ${ }^{2}$ Department of Oral Technology, School of Dentistry, University of Bonn, Bonn, Germany. ${ }^{3}$ Department of Oral and Craniofacial Health Sciences, College of Dental Medicine, University of Sharjah, Sharjah 27272, UAE. ${ }^{4}$ Research Institute of Medical and Health Sciences, University of Sharjah, Sharjah 27272, UAE. ${ }^{5}$ Department of Oral and Maxillofacial Surgery, Faculty of Dentistry, Suez Canal University, Ismaillia 41522, Egypt. ${ }^{6}$ Department of Oral Medicine and Radiology, Faculty of Oral and Dental Medicine, Future University in Egypt, Cairo, Egypt. ${ }^{7}$ Department of Orthodontics, Faculty of Oral and Dental Medicine, Future University in Egypt, Cairo, Egypt.

Received: 20 February 2021 Accepted: 9 May 2021

Published online: 13 May 2021

\section{References}

1. Ahmed HM. Different perspectives in understanding the pulp and periodontal intercommunications with a new proposed classification for endo-perio lesions. ENDO (Lond Engl). 2012;6:87-104.
2. Zakian CM, Taylor AM, Ellwood RP, Pretty IA. Occlusal caries detection by using thermal imaging. J Dent. 2010;38(10):788-95. https://doi.org/10. 1016/j.jdent.2010.06.010.

3. Saxena AK, Willital GH. Infrared thermography: experience from a decade of pediatric imaging. Eur J Pediatr. 2008;167(7):757-64. https://doi.org/10. 1007/s00431-007-0583-z.

4. Chan $\mathrm{CP}$, Lin CP, Tseng SC, Jeng JH. Vertical root fracture in endodontically versus nonendodontically treated teeth: a survey of 315 cases in Chinese patients. Oral Surg Oral Med Oral Pathol Oral Radiol Endod. 1999;87(4):504-7. https://doi.org/10.1016/s1079-2104(99)70252-0.

5. Gratt BM, Anbar M. Thermology and facial telethermography: Part II Current and future clinical applications in dentistry. Dento Maxillafac Radiol. 1998:27:68-74.

6. Gratt BM, Graff-Radford SB, Shetty V, Solberg WK, Sickles EA. A 6-year clinical assessment of electronic facial thermography. Dentomaxillofac Radiol. 1996:25:247-55.

7. Cummings M, Biagioni P, Lamey PJ, Burden DJ. Thermal image analysis of electrothermal debonding of ceramic brackets: an in vitro study. Eur J Orthod. 1991;21:111-8.

8. Tabatabaei N, Mandelis A, Amaechi BT. Thermophotonic lock-in imaging of early demineralized and carious lesions in human teeth. J Biomed Opt. 2011;16(7):071402. https://doi.org/10.1117/1.3564890.

9. Bonmarin M, Le Gal FA. Lock-in thermal imaging for the early-stage detection of cutaneous melanoma: a feasibility study. Comput Biol Med. 2014;47:36-43. https://doi.org/10.1016/j.compbiomed.2014.01.008.

10. Zhang H, Tavakolian P, Sivagurunathan K, Mandelis A, Shi W, Liu FF. Truncated-correlation photothermal coherence tomography derivative imaging modality for small animal in vivo early tumor detection. Opt Lett. 2019;44(3):675-8. https://doi.org/10.1364/OL.44.000675.

11. Sanchis-Sánchez E, Salvador-Palmer R, Codoñer-Franch P, Martín J, Vergara-Hernández C, Blasco J, Ballester E, et al. Infrared thermography is useful for ruling out fractures in paediatric emergencies. Eur J Pediatr. 2015;174(4):493-9. https://doi.org/10.1007/s00431-014-2425-0.

12. Hildebrandt C, Raschner C, Ammer K. An overview of recent application of medical infrared thermography in sports medicine in Austria. Sensors (Basel). 2010;10(5):4700-15. https://doi.org/10.3390/s100504700.

13. Zaproudina N, Varmavuo V, Airaksinen O, Närhi M. Reproducibility of infrared thermography measurements in healthy individuals. Physiol Meas. 2008;29(4):515-24. https://doi.org/10.1088/0967-3334/29/4/007.

14. Ammoush $M$, Gzawi $M$, Warawreh $A$, Hijazin $R$, Jafar $H$. Clinical evaluation of thermography as a diagnostic tool in oral and maxillo-facial lesions. JRMS. 2018:25(3):45-9. https://doi.org/10.12816/00532.

15. Yılmaz UN, Güneş Uysal RF, Dündar Yılmaz B, Tuncer MC. Histopathologic and immunohistochemical investigations of dental abscess formed in maxillofacial area. Folia Morphol (Warsz). 2019;78(4):754-61. https://doi. org/10.5603/FM.a2019.0051

16. Blomqvist A, Engblom D. Neural mechanisms of inflammation-induced fever. Neuroscientist. 2018;24(4):381-99. https://doi.org/10.1177/10738 58418760481.

17. Celeste CJ, Deschesne K, Riley CB, Theoret CL. Skin temperature during cutaneous wound healing in an equine model of cutaneous fibroproliferative disorder: kinetics and anatomic-site differences. Vet Surg. 2013:42(2):147-53. https://doi.org/10.1111/j.1532-950X.2012.00966.x.

18. Pauk J, Wasilewska A, Ihnatouski M. Infrared thermography sensor for disease activity detection in rheumatoid arthritis patients. Sensors (Basel). 2019;19(16):3444. https://doi.org/10.3390/s19163444.

19. Adam M, Ng EYK, Tan JH, Heng ML, Tong JWK, Acharya UR. Computer aided diagnosis of diabetic foot using infrared thermography: a review. Comput Biol Med. 2017;91:326-36. https://doi.org/10.1016/j.compb iomed.2017.10.030.

20. Komoriyama M, Nomoto R, Tanaka R, Hosoya N, Gomi K, lino F, et al. Application of thermography in dentistry-visualization of temperature distribution on oral tissues. Dent Mater J. 2003;22(4):436-43. https://doi org/10.4012/dmj.22.436.

\section{Publisher's Note}

Springer Nature remains neutral with regard to jurisdictional claims in published maps and institutional affiliations. 